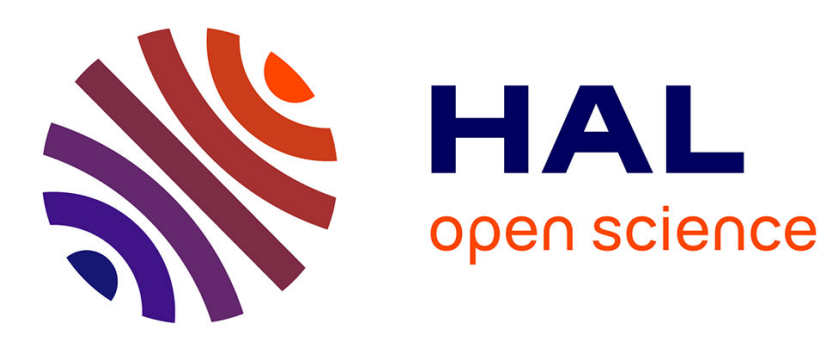

\title{
Bipolaronic charge density waves and polaronic spin density waves in the Holstein-Hubbard model
}

\author{
S. Aubry
}

\section{To cite this version:}

S. Aubry. Bipolaronic charge density waves and polaronic spin density waves in the Holstein-Hubbard model. Journal de Physique IV Proceedings, 1993, 03 (C2), pp.C2-349-C2-355. 10.1051/jp4:1993270 . jpa-00251353

\section{HAL Id: jpa-00251353 https://hal.science/jpa-00251353}

Submitted on 1 Jan 1993

HAL is a multi-disciplinary open access archive for the deposit and dissemination of scientific research documents, whether they are published or not. The documents may come from teaching and research institutions in France or abroad, or from public or private research centers.
L'archive ouverte pluridisciplinaire HAL, est destinée au dépôt et à la diffusion de documents scientifiques de niveau recherche, publiés ou non, émanant des établissements d'enseignement et de recherche français ou étrangers, des laboratoires publics ou privés. 


\title{
Bipolaronic charge density waves and polaronic spin density waves in the Holstein-Hubbard model
}

\author{
S. AUBRY
}

\author{
Laboratoire Léon Brillouin, CEA-CNRS, CE/Saclay, 91191 Gif-sur-Yvette cedex, France
}

\begin{abstract}
At large electron-phonon coupling, the phonon of the Holstein-Hubbard model can be (generally) treated classically. Exact results can be obtained for this model at any band filling and any finite dimension where the Hubbard term with coefficient $U$ is treated rigorously. The existence of chaotic bipolaronic states is proven when $U$ is smaller than a certain value $U_{\mathfrak{c}}$ and for an electron-phonon coupling $\mathrm{k}$ sufficiently large. The existence of magnetic polaronic states is also proven for positive $U$ and $k$ sufficiently large. For $0<U<U_{c}$, there exists an overlap region with chaotic mixed polaronic-bipolaronic states. There is a region for $U \approx \mathrm{U}_{\mathcal{C}} / 2$ and large $\mathrm{k}$ of special interest, where the bipolarons in their ground-state become singlet pairs of polarons bounded by a magnetic resonance. Then, the Peierls-Nabarro barrier which usually pins polarons and bipolarons to the lattice depresses to almost zero and the validity of the classical lattice approximation breaks down so that the initial exact results do not apply in that specific region. The formation of a superconducting state with possibly a high critical temperature, is then conjectured at a large coupling $\mathrm{k}$ in a narrow domain in-between the two insulating domains corresponding to Bipolaronic Charge Density Waves and to magnetic Polaronic Spin Density Waves respectively.
\end{abstract}

\section{Definition of the Model and Anti-Integrable Limit}

The Holstein-Hubbard model, which is obtained as a combination of the standard Holstein and Hubbard models, is likely the simplest model involving both an electron-phonon coupling and a direct electronelectron repulsion. Since it contains a minimum number of parameters, it is appropriate for understanding some basic mechanisms of the interplay between these interactions and for proving some rigorous results. Its Hamiltonian $\mathrm{H}=\mathrm{H}_{\mathrm{k}}+\mathrm{H}_{\mathrm{p}}+\mathrm{H}_{\mathrm{ep}}+\mathrm{H}_{\mathrm{u}}$

is the sum of

1- a tight binding Hamiltonian

$$
H_{k}=-\sum_{<i, j>\sigma} T c_{i, \sigma}^{+} c_{j, \sigma}
$$

describing a single electronic band with bandwidth $2 \mathrm{Td}$ where $\mathrm{T}$ is the transfer integral of the electrons between the neighbouring sites $<i, j>$ of a d-dimensional square lattice. $c_{i, \sigma}^{+}$and $c_{i, \sigma}$ are the standard creation and annihilation fermion operators of an electron at site $i$ with spin $\sigma=\uparrow$ or $\downarrow$ 2- a dispersionless phonon Hamiltonian

$$
H_{p}=\sum_{i}\left(\frac{p_{i}^{2}}{2 m}+\frac{1}{2} m \omega_{0}^{2} u_{i}^{2}\right)=\sum_{i} \hbar \omega_{0}\left(a_{i}^{+} a_{i}+\frac{1}{2}\right)
$$

of uncoupled quantum oscillators with mass $m$, stiffness constant $\kappa=m \omega_{0}^{2}$ and frequency $\omega_{0}$ at site $i$ where $u_{i}$ and $p_{i}$ are the conjugate position and momentum operators. $H_{p}$ can be also written with creation and annihilation boson operators $\mathrm{a}_{i}^{+}$and $\mathrm{a}_{i}$. 
3- a on-site electron-phonon coupling with coefficient $\mathrm{g}$ or $\lambda=\mathrm{g} \sqrt{2 \mathrm{~m} \omega_{0} / \mathrm{h}}$ (Holstein coupling)

$$
H_{e p}=g \sum_{i} n_{i}\left(a_{i}^{+}+a_{i}\right)=\lambda \sum_{i} n_{i} u_{i}
$$

which couples the electronic density operator $n_{i}=c_{i, \uparrow}^{+} c_{i, \uparrow}+c_{i, \downarrow}^{+} c_{i, \downarrow}=n_{i, \uparrow}+n_{i, \downarrow}$ with the local lattice distortion $\mathbf{u}_{\mathbf{i}}$

$$
\text { 4- a on-site repulsion term (Hubbard coupling) } \quad \mathrm{H}_{\mathrm{u}}=U \sum_{\mathrm{i}} \mathrm{n}_{\mathrm{i}, \uparrow} \mathrm{n}_{\mathrm{i}, \downarrow}=U \sum_{\mathbf{i}} \mathrm{c}_{\mathbf{i}, \uparrow}^{+} \mathrm{c}_{\mathrm{i}, \uparrow} \mathrm{c}_{\mathrm{i}, \downarrow}^{+} \mathrm{c}_{\mathrm{i}, \downarrow}
$$

with coefficient $U>0 . \lambda, \kappa$ and $U$ are classical constants which are not directly sensitive to isotope effects). It is now convenient to redefine new variables

$$
u_{n}=\frac{h \omega_{0}}{4 g}\left(a_{n}^{+}+a_{n}\right) \quad \text { and } \quad p_{n}=\frac{2 g}{h \omega_{0}} i\left(a_{n}^{+}-a_{n}\right)
$$

the commutator of which is $\left[u_{n}, p_{n}\right]=i$. In order to have only dimensionless parameters, we choose as a

$$
\begin{array}{ll}
\text { new energy unit } & \mathrm{E}_{0}=\frac{8 g^{2}}{h \omega_{0}} \\
\text { then Hamiltonian (1) becomes } & \hat{\mathrm{H}}=\frac{\mathrm{H}}{\mathrm{E}_{0}}=H_{A I}+t H_{K}+\beta H_{Q} \\
\text { where } & \mathrm{H}_{\mathrm{AI}}=\sum_{i}\left(\frac{1}{2}\left(\mathbf{u}_{\mathbf{i}}^{2}+\mathrm{n}_{\mathbf{i}} \mathbf{u}_{\mathbf{i}}\right)+v \mathrm{n}_{\mathrm{i}, \uparrow} \mathrm{n}_{\mathrm{i}, \downarrow}\right)
\end{array}
$$

with the coefficient

$$
t=\frac{T h \omega_{0}}{4 g^{2}}
$$

$t$ small is equivalent to a large electron-phonon coupling $k=2 g / \sqrt{T h \omega_{0}}=1 / \sqrt{t}$ in dimensionless units.

Finally, the term coming from the quantum lattice fluctuations is $\quad \mathrm{H}_{\mathrm{Q}}=\frac{1}{2} \sum_{\mathbf{i}} \mathbf{p}_{\mathrm{i}}^{2}$

with coefficient

$$
\beta=\frac{1}{4}\left(h \omega_{0} / 2 g\right)^{4}
$$

At large electron-phonon coupling $g>>h \omega_{0}$, this coefficient $\beta$ becomes small. (However, even small this term could play an essential role if the ground-state exhibits a gapless or almost gapless phonon softening or equivalently if the Peierls-Nabarro barrier vanishes or almost vanishes ${ }^{[1,2]}$ ). If $\beta$ is zero, operator $u_{i}$ commutes with the Hamiltonian and can be treated as a scalar (classical) variable. Parameter $t(6 \mathrm{~b})$ is not necessarily small because in most physical situations, we generally have $T>\aleph_{h} \omega_{0}$ (large electronic bandwidth). The standard theory of Charge Density Waves is developed within the adiabatic approximation (that is to take $\beta=0$ ). In that case, the model becomes variational. For a given set $\left\{\mathbf{u}_{\mathbf{i}}\right\}$, we formally calculate the ground-state with energy $\Phi_{\mathrm{el}}\left(\left\{\mathbf{u}_{\mathrm{i}}\right\}\right)$ of the many-body electron Hamiltonian

$$
H_{e l}=\sum_{i} \frac{1}{2} n_{i} u_{i}+v n_{i, \uparrow} n_{i, \downarrow}-\frac{t}{2} \sum_{<i, j>, \sigma} c_{i, \sigma}^{+} c_{j, \sigma}
$$

and we have to minimise the variational form

$$
\Phi\left(\left\{u_{i}\right\}\right)=\Phi_{e l}\left(\left\{u_{i}\right\}\right)+\sum_{i} \frac{1}{2} u_{i}^{2}
$$

which is the total of the elastic energy and of the electronic energy.

When the Hubbard term $v$ is zero, the eigen functions $\mid \Psi>$ of the electronic Hamiltonian (8a) correspond to a Slater determinant or equivalently can be written as

$$
\left|\Psi>=\prod_{v}\left(1-s_{v, \sigma^{+}}+s_{v, \sigma_{n, \sigma}}^{+}\right)\right| \varnothing>
$$


where $|\emptyset\rangle$ is the vacuum state. $s_{v, \sigma}$ is an occupation number which is 1 is the electronic state $v, \sigma$ is occupied and 0 if it is unoccupied. The new creation operators $c_{v, \sigma}^{+}=\sum_{n} \phi_{n}^{v} c_{n, \sigma}^{+}$

are defined with the normalised eigenstates $\phi_{n}^{v}$ of $\quad-t \Delta \phi_{n}^{v}+u_{i} \phi_{n}^{v}=E^{v}\left(\left\{u_{i}\right\}\right) \phi_{n}^{v}$

and $\Delta \phi_{\mathrm{n}}^{v}$ denotes the sum $\sum_{\mathrm{m}} \phi_{\mathrm{m}}^{v}$ for the sites m connected to $\mathrm{n}$. In that simpler situation,

$$
\Phi_{\mathrm{el}}\left(\left\{\mathbf{u}_{\mathrm{i}}\right\}\right)=\sum_{v} \sigma_{v} \mathbf{E}^{v}\left(\left\{\mathbf{u}_{\mathrm{i}}\right\}\right)
$$

where $\quad \sigma_{v}=\frac{1}{2}\left(s_{v}, \uparrow+s_{v, \downarrow}\right)=0,1 / 2$ or 1

Thus, when $v=0$ the electronic energy does not depend on the spin orientation

\section{Some Exact Results at Arbitrary Electronic Density .}

With no Hubbard term $(v=0)$, and when the electrons are supposed to be in their ground-state relatively to the lattice distortion ( that $\sigma_{v}=1$ for $E^{v}\left(\left\{u_{i}\right\}\right)<E_{F}$ and $\sigma_{v}=0$ for $E^{v}\left(\left\{u_{i}\right\}\right)>E_{F}$ where $E_{F}$ is the Fermi energy) it has been proved recently ${ }^{[3,4]}$ that when $t$ is not too large $\left(t<t_{b}\right)$, the variational form (8b) has infinitely many local minima corresponding to chaotic bipolaronic states and that the ground-state is one of them with a particular bipolaron ordering.

When the electrons are not in their ground-state relatively to the lattice distortion, this variational form has infinitely many other metastable states which corresponds physically to chaotic polaronic (for $t<t_{p}$ ) and mixed polaronic-bipolaronic states (for $t<t_{m}$ ). Of course, these new configurations are not groundstates but are excited states unless a magnetic field is applied to the system.

The magnetic field can be taken into account in Hamiltonian (1) by choosing

1- a transfer integral $T_{i j}=T \exp \left(i \theta_{i j}\right)$ in (2a) which depends on the bound $<i, j>$ by a complex phase $\theta_{i j}$ such that $\sum_{<i, j>\text { plaquette }} \theta_{\mathrm{ij}}=2 \pi \varphi$ where $\varphi$ is the number of quantum fluxes per plaquette (orbital effect) and 2- by adding to the electronic part (2a) a Zeeman term - h. $\left(n_{i, \uparrow}-n_{i, \downarrow}\right)$.

The theorems and their proofs for the existence of bipolaronic, polaronic and mixed polaronicbipolaronic are unchanged by the presence of a magnetic field, except that the bounds on $t$ are even increased. It has been proved that for a large enough magnetic field $h>h_{c}>0$, the ground state becomes a mixed polaronic-bipolaronic structure and depends on the magnetic field.

Exact bounds for $t_{b}, t_{p}$ and $t_{m}$ have been given in ref. 4 for the Holstein model on several lattices (cubic, triangular etc...) in 1,2 and 3 dimensions. For 1 and $2 \mathrm{~d}$ cubic lattices, these bounds approach rather well the order of magnitude of the bounds which can be observed numerically. For example, for the 1d Holstein model, these exact bounds are $t_{b}>0.181648$ for the purely bipolaronic states, $t_{p}>0.090824$ for the purely polaronic states and $t_{\mathrm{m}}>\mathbf{0 . 0 7 3 7 3 2}$ for the mixed polaronic bipolaronic states.

Exact properties were found for these ground-states and these metastable states. They are insulating structures with an electronic gap at the Fermi energy for the bipolaronic states. For the mixed polaronicbipolaronic states, singly occupied mid-gap electronic states appear. Their phonon spectrum also exhibits a strictly non vanishing gap which forbids the existence of solitons and gapless phason (in a strict sense). All these structures are "charge defectible" which means that the addition or the extraction of an electron or a pair of electrons to the whole structure, results only into some local variation of the electronic density which decays exponentially at infinity. This is the characteristic feature of a true insulator contrasting with the properties of metals and semi-conductors where extra charges delocalizes in the whole system. The characteristic length of decay physically correspond to the size of a bipolaron or of a polaron depending the type of excitation

The basic approach ${ }^{[3]}$ for finding these exact results, is to start from the limit $t=0 \quad(\beta=0)$, which we call "anti-integrable limit" by analogy with a similar limit which we introduced for dynamical systems ${ }^{[5,6]}$. By contrast with the usual band theory, which starts with perturbation expansions from delocalised electronic plane waves, we start our pertubation theory from the opposite limit where the electrons are localised on single sites. At this limit, Hamiltonian $H_{\text {AI }}$ commutes with electron density operators $n_{i, \uparrow}$ and $n_{i, \downarrow}$ at each site, so that for any arbitrary spatial distribution of the electrons, we have an eigen state. 
This electronic distribution can be characterized by a pseudo-spin distribution $\left\{\sigma_{i}\right\}$ with $\sigma_{i}=0$ for the unoccupied sites, $\sigma_{\mathrm{i}}=1 / 2$ for the singly occupied sites and $\sigma_{\mathrm{i}}=1$ for the doubly occupied sites. The variational form $(8 \mathrm{~b})$ becomes

$$
\sum_{i} \frac{1}{2}\left(\mathbf{u}_{i}+\sigma_{i}\right)^{2}-1 / 2 \sigma_{i}^{2}+2 \sigma_{i}\left(\sigma_{i}-1 / 2\right) v
$$

and is minimum for

$$
\mathbf{u}_{\mathrm{i}}=-\sigma_{\mathrm{i}}
$$

By definition, a bipolaron is a pair of localised electrons with opposite spins associated with a lattice distortion. A polaron is a localized single electron associated with a lattice distortion. A hole is a site without any electron. At the anti-integrable limit, the existence of bipolarons at sites $i$ where $\sigma_{i}=1$, of polarons at sites $i$ where $\sigma_{i}=1 / 2$ and of holes $\left(\sigma_{i}=0\right)$, is obvious. Our theorem (for $\left.v=0\right)$ consists in proving that when $t$ is switched to a non zero value, each of the local minima of the total energy (8b) (which does not depends on the spin orientation of the polarons) does not disappear and depends continuously on $t$ up to an upper bound.

For $v \neq 0$, our theorems can be partially extended when considering the electrons in the ground-state of the many-body Hamiltonian (8a). For that purpose, it is necessary to choose a chemical potential $\mu$, in order that at the limit $t=0$, the ground-state of the local electron Hamiltonian

$$
H_{i}=-\left(n_{i, \uparrow}+n_{i, \downarrow}\right)\left(\frac{\sigma_{i}}{2}+\mu\right)+v n_{i, \uparrow} n_{i, \downarrow}
$$

be really obtained for $\frac{1}{2}\left(n_{i, \uparrow}+n_{i, \downarrow}\right)=\sigma_{i}$. The choice of $\mu$ turns out to be not always possible. More precisely, after a complete inspection of (12a), it comes out that this choice is possible if and only if

$1-v<\frac{1}{2}$ for the purely bipolaronic structures (where $\sigma_{\mathrm{i}}=0$ or 1 )

2- $0<v$ for the purely polaronic structures (where $\sigma_{\mathrm{i}}=0$ or $1 / 2$ )

3- $0<v<\frac{1}{2}$ for the mixed bipolaronic polaronic structures (where $\sigma_{\mathrm{i}}=0,1 / 2$ or 1 ).

We proved very recently ${ }^{[7]}$ the existence of non vanishing bounds $t_{b}$ and $t_{p}$ depending on $v$ such that the total energy (8b) has at least one local minima $\left\{u_{i}\right\}$ converging to $\left\{-\sigma_{i}\right\}$ for $t \rightarrow 0$

1 - for any bipolaronic structure characterized by $\left\{\sigma_{\mathrm{i}}=0\right.$ or 1$\}$, when $v<\frac{1}{2}$ and $t<t_{b}$,

2- for any polaronic structure characterized by $\left\{\sigma_{i}=0\right.$ or $\left.1 / 2\right\}$, when $0<v$ and $t<t_{p}$,

3 -for any mixed bipolaronic polaronic structures characterized by $\left\{\sigma_{i}=0,1 / 2\right.$ or 1$\}$ when $0<v<\frac{1}{2}$ and $\mathrm{t}<\min \left(t_{b}, t_{p}\right)$

These bounds $t_{b}$ and $t_{p}$ has not been yet optimized. The figure shows the shape of the obtained domains of proven existence of bipolaronic, polaronic and mixed structures.

Although this theorem is not as much precise as in the case $v=0$, it is already interesting because it proves

1- the persistence of bipolaronic states up to $v<1 / 2$ (and not beyond) (and for negative $v$ )

2- that the polaronic states only exists for $v>0$

3 - that there exists a complex intermediate region with mixed polaronic bipolaronic states for $0<v<1 / 2$

We have not proven the unicity of this state as in the case $v=0$. This property is likely true for the bipolaronic states but might be wrong for the mixed polaronic bipolaronic states and the purely polaronic states. Unlike for the bipolaronic structures, the excitation spectrum of the polaronic and mixed polaronic bipolaronic might become gapless. Let us explain why:

At the anti-integrable limit, the spin degrees of freedom of the polarons are totally degenerate. Unlike the case $v=0$ where the spins remain uncoupled, these spins couple (by exchange integrals) as soon $t$ is not zero. If the dimensionality of the model is sufficient, the spin degeneracy could be raised and a resulting magnetic structure with many metastable states should be expected on top of the distribution of polarons (and bipolarons). It should be similar to a spin glass system if the underlying polaronic distribution is random. Nevertheless, the invariance under a global rotation of the spins is preserved, so that the magnon excitations are gapless according to the well-known Goldstone theorem. For that reason, the excitation spectrum of these states might be gapless. In other situations especially in low dimensional system, the polaronic or mixed polaronicbipolaronic structures, could be non magnetic. This is for example the 
situation for the spin resonant bipolaron described in the next section, where the magnetism of single polarons vanishes by the formation of a singlet state.

\section{Two electrons in the Holstein-Hubbard model :Spin Resonant Bipolarons. Conjecture about high $T_{c}$ superconductivity}

There is a region of special interest corresponding to the case $v=1 / 4$ which means in original units

$$
g \approx \sqrt{\frac{U \hbar \omega_{0}}{2}}
$$

where for $t=0$, the energy of a bipolaron $-1 / 2+v$ given by (11a) for is equal to the energy $-2 \times 1 / 8$ of two polarons. Let us note that in the anti-adiabatic regime $g<<h \omega_{0}$, the electron phonon interaction can be reduced via a unitary transformation (Lang-Firsov) to an effective attractive electron-electron interaction $-2 \mathrm{~g}^{2} / \mathrm{h} \omega_{0}$ between the electrons. When (13) is exactly fulfilled, this attractive interaction exactly cancels the direct electron-electron interaction U. Then, an ideal Fermi liquid should be restored for the eigenstates of the Holstein-Hubbard model. In fact, we consider here the adiabatic regime where $\mathrm{g} \gg \mathrm{h}_{0}$ where the effective attractive electron-electron interaction due to the phonons is drastically retarded and cannot cancel the direct intantaneous interaction. It is clear that a Fermi liquid cannot be recovered in that regime.

In fact at the adiabatic limit, the spins of the polarons interacts anti-ferromagnetically via an exchange integral as soon as $t \neq 0$. There is a binding energy between two polarons due to the formation of a singlet state $1 / \sqrt{2}$ ( $|\uparrow \downarrow\rangle+|\downarrow \uparrow\rangle$ ) with their spins while the triplet states are antibounding. This binding energy which originates from the exchange interaction between the spins of the polarons becomes the predominant term in the regime defined by (13). Indeed, it has been easily numerically observed for a single pair of electrons in the one dimensional Holstein-Hubbard model ${ }^{[8]}$ that the minimum energy for two polarons is not obtained when they are localized on the same site but becomes pair of polarons on neighbouring sites. This binding energy is of the order of the exchange integral $\mathrm{J}$ between the spins of the polarons which of course depends on their distance. When the Hubbard term becomes larger and condition (13) is no more fulfilled, the distance between the polarons of the pair increases and their binding energy weakens.

Let us remark that this spin resonant bipolaron (SRB) have some similarities with the Resonant Valence Bound (RVB) state ${ }^{[9]}$ of two electrons which was proposed some years ago by P.Anderson for the pure Hubbard model. In fact, this state does not exist in this model but it requires the interplay of both a strong enough electron-phonon coupling (close to the adiabatic limit) and a strong Hubbard term fulfilling approximately condition (13).

Let us explain now why we expect that the existence of SRB, should be more favorable for high $\mathrm{T}_{\mathcal{c}}$ superconductivity than the standard bipolarons obtained at large electron-phonon coupling without Hubbard term.

First let us note that no superconductivity can be obtained within the adiabatic approximation $(\beta=0)$. At low electron-phonon coupling $\mathrm{g}<\sqrt{\mathrm{T} h \omega_{0}}$, the standard BCS theory applies. The critical temperature depends roughly on $k=2 \mathrm{~g} / \sqrt{\mathrm{T} h \omega_{0}}$ as $h \omega_{0} \exp \left(-\propto / \mathrm{k}^{2}\right)$ is restricted to relatively small values because $\mathrm{k}$ has to be smaller than 1 . At larger electron phonon coupling, electrons certainly forms bipolarons at any electronic densities ${ }^{[5]}$. Theories of bipolaronic superconductivity ${ }^{[10]}$ were proposed with no direct electron-electron repulsion $(v=0)$ but fail in practice for the reason that in real system $\beta$ is generally small although not zero. Then, the bipolaronic superconductivity is disfavored because the effective mass of the bipolarons exponentially diverges as soon as the electron phonon coupling $\mathrm{g}$ increases beyond the $\mathrm{BCS}$ regime $(k<\approx 1$ or $t \approx>1)$. Equivalently, the coherence energy (or band width) between these bipolarons (which are hard core bosons) which would determine the critical superconducting temperature, drops down to zero and becomes negligible which confirms the validity of the adiabatic approximation in that regime ${ }^{[1,2]}$. The potential interactions between these bipolarons are in fact the major terms of the Hamiltonian (in real systems, there is also Coulomb and elastic interactions between the bipolarons not taken into account in our model). A spatial and insulating ordering of bipolarons appears instead of this 
speculative bipolaronic superconductivity, as predicted by our theorem on the ground-state ${ }^{[5]}$ at $\beta=0$ and $v=0$.

Therefore, the major challenge of high $\mathrm{Tc}$ superconductivity is to find a physical mechanism which restore a large enough coherence energy for the bipolarons (bosons) in order to prevent the system from a spatial ordering and to favor a superfluid state. The origin of the collapse of the coherence energy of the bipolarons at large electron-phonon coupling in most models, is essentially due to the sharp increase of the Peierls-Nabarro barrier. The Peierls-Nabarro barrier of a bipolaron is by definition the minimum of the extra energy required for moving adiabatically a bipolaron from a given site to a neighbouring site. When the quantum lattice fluctuations are taken into account, higher is this barrier lower will be the tunnelling (or coherence) energy of a bipolaron through this barrier (for $\beta$ small ). For increasing this coherence energy which potentially determines the critical temperature of the possible superfluid bipolaronic state, one has to reduce the Peierls-Nabarro barrier of the bipolarons as much as possible. Then, with rather small quantum lattice fluctuations $\beta$, the superfluid state of bipolarons could become the ground-state. The middle state of the Peierls-Nabarro barrier of a bipolaron is a pair of polarons on two adjacent sites. If the Hubbard term $v$ is increased from zero, this state become energetically more favorable so that the Peierls-Nabarro barrier is depressed. There is a critical point for $v$ at which the energy difference between the middle of the potential barrier and the bottom of this potential just vanishes. In fact, the Peierls Nabarro barrier does not strictly vanish because its top is no longer the middle state but in any case, it is sharply depressed as observed numerically in [8]. This point just corresponds to condition (13) at the anti-integrable limit.

In summary, our exact results confirm the interest of a perturbation approach from the "anti-integrable" limit of localised electrons (the "chemist limit") which is a limit opposite to the most used band limit of delocalised electrons. It reveals wide domains of parameters with insulating bipolaronic and polaronic phases which are relevant for understanding the real Charge Density Waves and Spin Density Waves respectively. Many questions concerning the detailed structure of the phase diagram are opened but our analysis reveals also an intermediate narrow but specially interesting although questionable region in between these two phases where at the adiabatic limit, we should have mixed polaronic bipolaronic states. In that region, the structure of the stable bipolarons change. They becomes SRB which are pairs of polarons bounded by a spin resonance (singlet states) (the distinction between SRB and standard bipolarons is obvious at the anti-integrable limit). These SRB are weakly pinned to the lattice so that they should be highly sensitive to the effect of quantum lattice fluctuations. The occurence of SRB superconductivity which could reach unusually high temperature is conjectured in that specific region. 


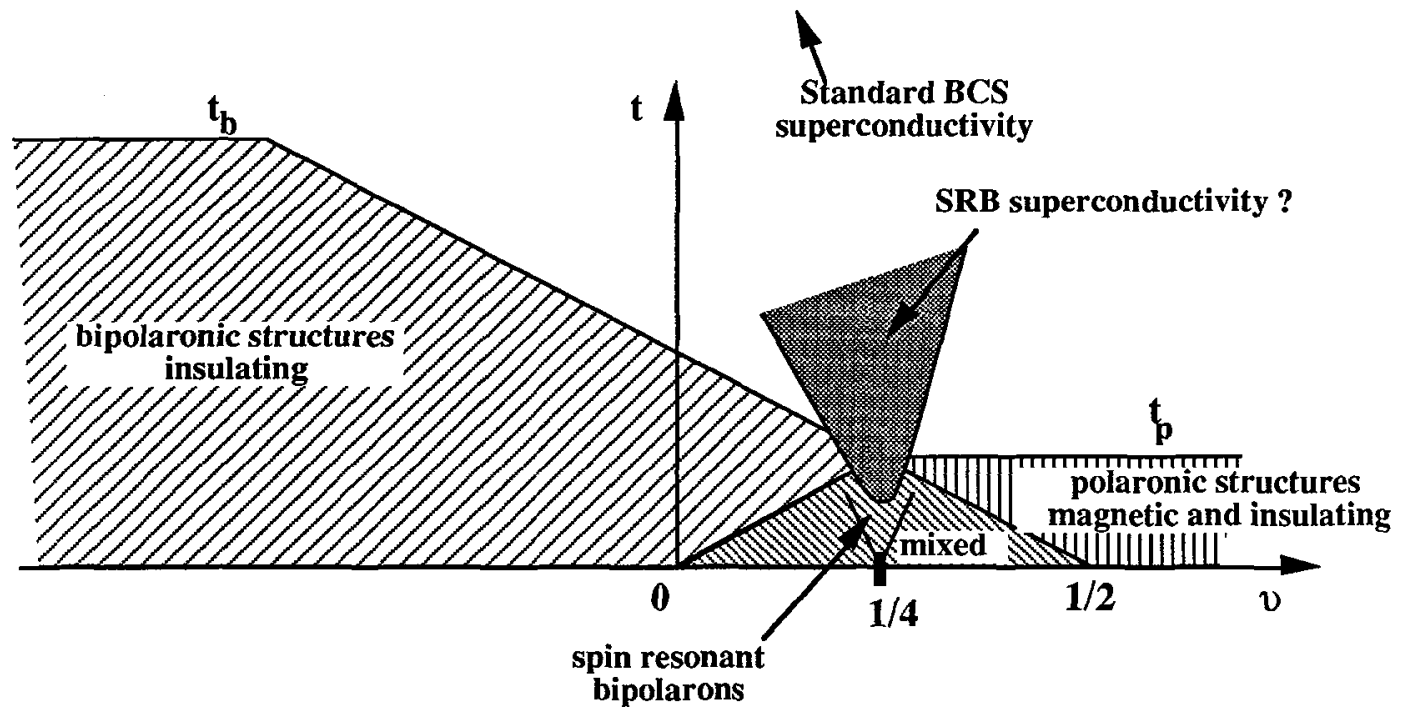

Figure: Scheme of the minimum domains of existence for the bipolaronic, polaronic and mixed polaronic-bipolaronic structures in the adiabatic Holstein Hubbard model (at the present stage of our work). Starting from the point $\mathrm{t}=0, \mathrm{v}=1 / 4$, there is a domain (not yet accurately determined) where the bipolarons in their ground-state are pairs of polarons bounded by a spin resonance. In that region the validity of the adiabatic approximation breaks down for $\beta$ rather small and the quantum lattice fluctuation could produce a superconducting phase possibly with a high $T_{c}$. . (The tongue shape of this superconducting region is speculative.)

[1] S.Aubry, G. Abramovici, D.Feinberg, P. Quemerais and J.L. Raimbault in Non-Linear Coherent Structures in Physics, Mechanics and Biological Systems (Lect. Notes in Physics, Springer vol 353 p.103-116 (1989)

[2] S.Aubry in Microscopic Aspects of Non-Linearity in Condensed Matter Physics ed. A.R.Bishop, V.L.Pokrovsky and V.Tognetti NATO ASI Series, Series B vol 264 Plenum p.105-114 (1991)

[3] S.Aubry, G.Abramovici and J.L. Raimbault Chaotic Polaronic and Bipolaronic States in the Adiabatic Holstein Model J. of Stat.Phys. 67 (1992) 675-780

[4] C.Baesens and R.MacKay Improved proof of existence of chaotic polaronic and bipolaronic states for the adiabatic Holstein model and generalisations pre-print (1992)

[5] S. Aubry and G. Abramovici Chaotic Trajectories in the Standard Map, The concept of AntiIntegrability Physica D43 (1990) 199-219 S.Aubry The Concept of Anti-Integrability: Definition, Theorems and Applications in "Twist Mappings and their Applications" eds. Richard McGehee and Kenneth R. Meyer, The IMA Volumes in Mathematics and Applications 44 (1992) 7-54 (Springer)

[6] S.Aubry The Concept of Anti-Integrability Applied to Dynamical Systems and to Structural and Electronic Models in Condensed Matter Physics submitted to Physica D special issue ed.J.Meiss (1993)

[7] S.Aubry in preparation

[8] S.Aubry Bipolaronic Charge Density Waves, Polaronic Spin Density Waves and High Tc Superconductivity in Phase Separation in Cuprate Superconductors ed. KA Müller and G.Benedek World Publishing Cie (The science and culture series-Physics) (1992) 304-334

[9] P.Anderson Science 2351196 (1987) P.Anderson, G.Baskaran, Z.Zou and T.Hsu Phys.Rev.Letts. 58 2790 (1987)

[10] A.S.Alexandrov, J. Ranninger and S. Robaszkiewicz Phys.Rev. B33 p.4526 (1986) 